\title{
Factores de riesgo y secuelas reproductivas asociados a la infección por Chlamydia trachomatis en mujer es infértiles
}

\author{
Fernando Guerra-Infante, Dr en $C_{1}^{(1-2)}$ Saúl Flores-M edina, M en $C_{1}{ }^{(1)}$ \\ Gabriel Arteaga-Troncoso, $\mathrm{Dr}$ en $\mathrm{C}_{\text {, }}^{(1)}$ Angélica Zamora-Ruiz, $M$ en $C$, ${ }^{(1)}$ \\ Marcela López-H urtado, M en C, ${ }^{(1)}$ Federico Javier 0 rtiz-Ibarra, MC.(1)
}

\begin{abstract}
Guerra-Infante F, Flores-Medina S, Arteaga-Troncoso G, Zamora-Ruiz A, López-Hurtado M, Ortiz-Ibarra FJ.

Factores de riesgo y secuelas reproductivas asociados a la infección por Chlamydia trachomatis en mujeres infértiles.

Salud Publica Mex 2003;45 supl 5:S672-S680. El texto completo en inglés de este artículo está disponible en: http://www.ínsp.mx/salud/index.html
\end{abstract}

\section{Resumen}

O bjetivo. Comparar la información clínica y el estilo de vida sexual en dos grupos de mujeres con y sin infección por C hlamydia trachomatis que asisten a la clínica de infertilidad del Instituto N acional de Perinatología, de la Ciudad de México. Material y métodos. De febrero a noviembre de 1998, se realizó un estudio prospectivo en pacientes con diagnóstico de infertilidad. En el estudio se incluyó a pacientes con diagnóstico de infertilidad, tratados en el Instituto $\mathrm{N}$ acional de Perinatologia, de la Ciudad de México, durante 1988. Las muestras endocervicales de 309 mujeres, que incluyeron a 77 con infección y a 232 sin infección, fueron examinadas para Chlamydia trachomatis, usando inmunofluorescencia directa. Los cultivos vaginales fueron obtenidos antes de iniciar el tratamiento.También se investigó la presencia de otros agentes infecciosos de transmisión sexual y la información demográfica, de conducta sexual, histórica y clínica fue recopilada de cada paciente. Los datos clínicos y ginecológicos de ambos grupos fueron comparados por $\chi^{2}$. La magnitud de las asociaciones fueron establecidas por razón de momios en análisis bivariados. Se realizó un análisis de regresión logística para establecer los efectos confusores en relación con los factores analizados. Las diferencias fueron consideradas estadísticamente significativas si $p<0.05$. Resultados. Para el estudio 309 mujeres fueron elegibles, 77 (24.9\%) cursaron con infección por C trachomatis, de éstas,
Guerra-Infante F, Flores-Medina S,

Arteaga-Troncoso G, Zamora-Ruiz A, López-Hurtado M, Ortiz-lbarra FJ.

Risk factors and reproductive sequelae associated with Chlamydia trachomatis infection in infertile women.

Salud Publica Mex 2003;45 suppl 5:S672-S680.

The English version of this paper

is available at: http://www.insp.mx/salud/index.html

\section{A bstract}

0 bjective. To assess the clinical and sexual lifestyle characteristics associated with Chlamydia trachomatis infection among women diagnosed with infertility. Material and Methods. Study subjects were women with an infertility diagnosis attending the infertility clinic at the $\mathrm{N}$ ational Institute of Perinatology. Endocervical specimens from 309 women were examined for Chlamydia trachomatis using the direct immunofluorescence method.Vaginal culture samples were taken before initiating treatment. The presence of other sexually-transmitted infections was also assessed. Demographic, sexual behavioral, historical, and clinical data were collected for each subject. Results. Seventy-seven women (24.9\%) were infected with $\mathrm{C}$ trachomatis. Of these women, $70(90.9 \%)$ had only one sexual partner, 58 (75.3\%) were married, and $19(24.7 \%)$ were single or had a common-law sex partner. Intrauterine device (IUD) was the main contraceptive method used in 15 women (19.5\%). A coinfection with other sexually-transmitted microorganisms was detected in 41 of these patients (53.2\%). N ineteen (24.7\%) women had undergone tubal ligation. Among women with C trachomatis infection, reproductive sequelae were found: $24(31.2 \%)$ women had had abortions and 50 (64.9\%) had had a miscarriage. In 26 women cervical abnormalities were detected (33.8\%); 50 (64.9\%) had purulent vaginal discharge and 14 (18.1\%) had pelvic pain. Bivariate

(1) Departamento de Infectología e Inmunología Perinatal del Instituto $N$ acional de Perinatología. México, DF, México.

(2) Departamento de Microbiología de la Escuela N acional de Ciencias Biológicas del Instituto Politécnico N acional. México, D F, México.

Fecha de recibido: 29 de mayo de 2002 - Fecha de aprobado: 22 de noviembre de 2002 Solicitud de sobretiros: Dr en C. Fernando M G uerra Infante. Departamento de Infectología e Inmunología Perinatal del Instituto N acional de Perinatología. Montes U rales 800, Colonia Lomas de Virreyes 11000, México, DF. Correo electrónico: fguerrai@ sni.conacyt.mx 
$70(90.9 \%)$ mencionaron tener un compañero sexual, 58 (75.3\%) estaban casadas, 19 (24.7\%) eran solteras o vivían en concubinato, 15 (19.5\%) utilizaron el dispositivo intrauterino para el control de la natalidad, 41 (53.2\%) presentaron coinfección con otros agentes infecciosos de transmisión sexual, y 19 (24.7\%) cursaron con infertilidad por obstrucción tubárica. Las secuelas reproductivas observadas en las pacientes con infección por C trachomatis mostraron que 24 (31.2\%) tuvieron abortos, 50 (64.9\%), gestaciones previas, 26 (33.8\%) anormalidades en el cérvix y $50(64.9 \%)$ secreciones vaginales purulentas, con dolor (18.1\%). El análisis bivariado mostró que los factores de riesgo asociados significativamente con la infección por $\mathrm{C}$ trachomatis fueron la presencia de un compañero sexual ( $\mathrm{R}=2.96$, IC 95\% 1.22-7.5, $p=0.008$ ), concubinato ( $R M=3.68, p=0.03)$ y uso de dispositivo intrauterino ( $R M=2.42, p=0.01)$. C onclusiones. C onsistentemente, la alta prevalencia de la infección por $\mathrm{C}$ trachomatis ocurre en las mujeres mexicanas con infertilidad. Los resultados sugieren que la infección por $\mathrm{C}$ trachomatis está aso ciada a la convivencia con una pareja sexual, al estado civil y al uso de dispositivo intrauterino como método de control natal. La presencia de ectropión y friabilidad a la exploración obstétrica del cérvix deberán ser consideradas como predictores en la búsqueda intencionada de $\mathrm{C}$ trachomatis. La identificación y el tratamiento presuntivo deberán ser evaluados como medidas de prevención y control para la infección por C trachomatis entre las mujeres reconocidas en riesgo. El texto completo en inglés de este artículo está disponible en: http://www.insp.mx/salud/index.html

Palabras clave: Chlamydia trachomatis; factores de riesgo; infertilidad; obstrucción tubárica; México analysis revealed that the risk factors for $\mathrm{C}$ trachomatis infection in women with infer tility were: having a sex partner $(0 \mathrm{R}=2.96,95 \% \mathrm{Cl} 1.22-7.5, p=0.008)$, common-law union $(0 R=3.68, p=0.03)$, and IUD use $(0 R=2.42, p=0.01)$. Conclusions. A consistent relationship between $\mathrm{C}$ trachomatis infection and infertility was found. Infection with $\mathrm{C}$ trachomatis in women with infertility was associated with having one single sexual partner, marital status, and IUD use. The presence of ectropion and friability of the cervix may signal $C$ trachomatis infection. Identification and presumptive therapy should be evaluated as measures to prevent and control $\mathrm{C}$ trachomatis infection in patients at risk.The English version of this paper is available at: http://www.insp.mx/salud/index.html

Key words: Chlamydia trachomatis; fallopian tube occlusion; infertility; risk factors; Mexico os datos recopilados por la Organización Mundial L de la Salud señalan que cerca de 89 millones de personas presentan infección por Chlamydia trachomatis; sólo en Estados Unidos de América (EUA) se reportan cada año más de cuatro millones de casos nuevos, de los cuales 2.6 se presentan en mujeres, 1.8 en varones y 250000 en recién nacidos. ${ }^{1,2}$

La infección endocervical por C trachomatis es un problema de salud pública de la mujer en edad reproductiva. Publicaciones recientes refieren que entre 70 y $90 \%$ de estas pacientes son asintomáticas, y que la infección puede persistir por meses o años. ${ }^{1,2}$ Cuando las pacientes no son identificadas ni tratadas se permite que el agente infeccioso invada los tejidos causando procesos patológicos como la enfermedad pélvica inflamatoria (EPI); además, se ha asociado con otros padecimientos como la infertilidad por obstrucción tubárica (OTB), embarazos ectópicos, artritis reactiva y endocarditis. $^{3,4}$

Algunos estudios seroepidemiológicos han descrito que una proporción de 40 a $80 \%$ de los casos de EPI y de OTB son debidos a la infección por C trachomatis, ${ }^{5,6}$ es por ello que la detección primaria y el tratamiento de estas infecciones asintomáticas son necesarios e indispensables para evitar las secuelas que produce su presencia.

Se han identificado diversos factores de riesgo asociados con la historia natural de la infección por $C$ trachomatis: el número de parejas sexuales, la edad, el nivel socioeconómico, los hábitos higiénicos, el empleo de anticonceptivos orales y la presencia de otras infecciones de transmisión sexual, ${ }^{7-10}$ sin embargo, esta información se ha descrito para poblaciones femeninas con diferentes hábitos y costumbres a las de la mujer mexicana.

Por otra parte, la información epidemiológica ha documentado la influencia que tienen los factores genéticos y socioculturales sobre la susceptibilidad que pueden presentar las mujeres y hombres a la infección por C trachomatis. En México, aún se desconoce la prevalencia de la infección y sólo algunos reportes sugieren una frecuencia de 4 a 10\% en población abierta, de 
$16 \%$ en pacientes que asisten a la clínica de infecciones de transmisión sexual y de $21 \%$ en pacientes diagnosticadas con EPI, empleando pruebas de laboratorio como cultivo celular, inmunofluorescencia directa y técnicas moleculares. ${ }^{11-14}$ De igual manera, se desconocen los factores de riesgo inherentes al desarrollo de la infección en la población femenina mexicana, así como la relación entre la infección y las anormalidades del cérvix durante la exploración física. Por lo tanto, el objetivo de esta investigación fue contrastar los datos clínicos y del estilo de vida sexual en dos grupos de pacientes con y sin aislamiento de $C$ trachomatis en una población de mujeres que asisten a la clínica de infertilidad del Instituto Nacional de Perinatología, de la Ciudad de México.

\section{Material y métodos}

Se efectuó un estudio abierto que incluyó a todas las pacientes que asistieron a la clínica de infertilidad del Instituto Nacional de Perinatología, de febrero a noviembre de 1998. El desarrollo del estudio consistió en una serie de visitas para: a) interrogatorio médico, b) consentimiento informado y c) toma de las muestras cervicovaginales. Fueron elegibles todas las pacientes con antecedentes de infertilidad (primaria o secundaria), mayores de 18 años de edad con vida sexual activa. Se llevó a cabo la toma de las muestras endocervicales, previo consentimiento informado, y la obtención de la información médica a partir de los expedientes clínicos. Se evaluaron como variables predictivas un conjunto de factores potenciales que de una $u$ otra forma reflejaron las condiciones del estilo de vida sexual de las pacientes, tales como: edad, estado civil (casada, soltera, concubinato), métodos de regulación de la fertilidad (sin control, hormonal, barrera, natural), número de compañeros sexuales $(1 \mathrm{o} \geq 2)$ y la presencia de otros agentes causantes de infecciones de transmisión sexual e infertilidad por obstrucción tubárica; así como la información ginecológica para identificar apariencia del cérvix (normal, ectropión, friabilidad, eritema), presencia de descarga vaginal, con o sin dolor, $\mathrm{y}$ antecedentes de abortos y gestaciones previas. Las pacientes fueron excluidas de la investigación por las siguientes razones: a) que se hubieran aplicado duchas vaginales previas a la toma de la muestra cervical; b) que hubiesen recibido terapia antimicrobiana sistémica o local 30 días previos a la evaluación, y c) que hubieran tenido relaciones sexuales 48 horas antes de la obtención de la muestra. Se definió el término infertilidad como la incapacidad temporal o definitiva de la pareja sexual para concebir un producto viable des- pués de un tiempo determinado de relaciones sexuales sin control voluntario.

Muestras endocervicales. La toma de la muestra se realizó empleando dos hisopos de algodón estériles y un tercero, de alginato de calcio. Los dos primeros fueron utilizados para el cultivo microbiológico en diferentes medios artificiales, y el tercero fue depositado en el medio de transporte 2SP para el cultivo de $\mathrm{C}$ trachomatis.

Aislamiento microbiológico. Cada una de las muestras endocervicales fue cultivada en medios especiales para el aislamiento de los siguientes microorganismos: Neisseria gonorrhoeae, Gardnerella vaginalis, Candida albicans, Ureaplasma urealyticum, Mycoplasma hominis, Streptococcus agalactiae.

Aislamiento de Chlamydia trachomatis por cultivo celular. El diagnóstico de $C$ trachomatis se llevó a cabo por cultivo en células McCoy y HeLa-299 como previamente fue descrito por nuestro grupo. ${ }^{15}$ En general, se adicionaron $1 \times 10^{6}$ de estas células a microplacas de 24 pozos de fondo plano que contenían cubreobjetos de $13 \mathrm{~mm}$ de diámetro. La placa fue incubada a $37^{\circ} \mathrm{C}$, durante $24 \mathrm{~h}$, en incubadora de $\mathrm{CO}_{2}$. $\mathrm{Al}$ término de la incubación se eliminó el sobrenadante y en cada pozo se adicionaron 300 microlitros $(\mathrm{ml})$ de la muestra clínica. La placa fue centrifugada a $3000 \mathrm{rpm}$ por $1 \mathrm{~h}$ a temperatura ambiente. Se desechó el sobrenadante y se adicionó $1 \mathrm{ml}$ de medio mínimo esencial suplementado con suero fetal de ternera a $10 \%$, glucosa a $5 \%$ y cicloheximida a una concentración final de 1 microgramo $(\mathrm{mg}) / \mathrm{ml}$. La placa se incubó, por 72 h, a $37^{\circ} \mathrm{C}$ en atmósfera de $\mathrm{CO}_{2}$ a $5 \%$. Los cubreobjetos se fijaron con metanol, para realizar el diagnóstico de Chlamydia trachomatis mediante la identificación de cuerpos elementales por la técnica de inmunofluorescencia directa.

Identificación de Chlamydia trachomatis por inmunofluorescencia directa. Los cubreobjetos, obtenidos del cultivo celular, fijados con metanol, fueron teñidos mediante la adición de $20 \mathrm{ml}$ de anticuerpos monoclonales fluoresceinados, anti-proteína principal de la membrana externa de $C$ trachomatis, confirmatorio para cultivo celular (Syva Mycrotrak, Syva Company. Palo Alto, CA, EUA). La muestra se incubó por 30 minutos en cámara húmeda a temperatura ambiente. Los cubreobjetos se lavaron exhaustivamente con agua desionizada para eliminar el exceso de reactivo; se permitió el secado de la muestra y se adicionó el líquido de montaje. Los cubreobjetos fueron observados en el microscopio de epifluorescencia (Axiovert 100, Carl Zeiss) para la identificación de cuerpos elementales (partícu- 
las extracelulares) e inclusiones (cuerpos reticulares) de aspecto de color verde manzana. Se consideraron muestras positivas cuando se hallaron uno o más cuerpos elementales o inclusiones.

Análisis estadístico. La muestra de 309 observaciones permitió subdividir en dos grupos de tratamiento a las pacientes: un grupo, con identificación positiva a $C$ trachomatis, y otro grupo testigo, sin aislamiento. Los datos clínicos y ginecológicos observados en ambos grupos fueron comparados por la prueba de ji-cuadrada. La magnitud de las asociaciones entre las variables es expresada como la razón de momios (RM) y su valor del intervalo de confianza de $95 \%$, en análisis bivariados. El análisis de tendencia para proporciones fue usado para establecer el nivel de riesgo en las variables. Se adoptó modelo de regresión logística múltiple, para establecer el efecto confusor de la edad con la relación a los factores analizados. Las diferencias fueron consideradas estadísticamente significativas si $p<0.05$. La información obtenida fue analizada con el programa SPSS para Windows (versión 10, 1999).

\section{Resultados}

En el cuadro I se presenta la información referida a los datos clínicos y microbiológicos de la población estudiada. Un total de 309 mujeres infértiles, con un promedio de edad de $29.8 \pm 5.3$ años, fueron incluidas para el estudio; $24.9 \%$ tuvieron aislamiento de $C$ trachomatis y de éstas, $71.4 \%$ fueron obtenidas en el grupo de edad de 25 a 34 años (mediana de 30, rango de 17 a 48 años).

Con relación al número de compañeros sexuales, $22.7 \%$ de las pacientes con infección respondieron tener al menos una pareja, y con más de una $2.3 \%$. En las pacientes sin aislamiento, $57.9 \%$ mencionó que la relación sexual había sido con una pareja y $17.2 \%$ con más de una. La frecuencia de aislamiento en las pacientes con un compañero sexual se asoció significativamente $(\mathrm{RM}=2.96$, IC 95\% 1.22-7.5; $p=0.008)$.

Se indagó sobre el estado civil de las pacientes con infección; de ellas, 75.3\% (58/77 aislamientos), refirieron ser casadas, $2.6 \%$ solteras y $22.1 \%$ vivir en concubinato. La probabilidad de aislamiento en las pacientes que refirieron convivir en concubinato fue 3.7 veces mayor que en las pacientes solteras $(p<0.05)$.

Asimismo, se identificaron los métodos de regulación de la fertilidad en las pacientes con infección; los datos muestran que en $50.6 \%$ el aislamiento fue obtenido de quienes refirieron no tener algún método anticonceptivo. Los métodos hormonales fueron empleados en $15.6 \%$ de las pacientes con infección. Cierta tendencia en el aislamiento se observó en las pacientes que utilizaron dispositivo intrauterino (DIU); la posibilidad de la infección fue 2.4 veces más que en las pacientes sin método de control $(p<0.01)$.

En las mujeres con aislamiento de $C$ trachomatis la identificación microbiológica de otros agentes infecciosos fue obtenida en las muestras cervicales en $54.7 \%$ de los casos (41/77); la candidiasis y la vaginosis bacterianas estuvieron presentes, y en los cuales la coinfección con Candida albicans fue observada en 37.7\% (RM= 1.15), y con Gardnerella vaginalis, en $15.6 \%$ ( $R M=0.47$, $p>0.05)$. El análisis laparoscópico en las pacientes con infección por C trachomatis evidenció que $75.3 \%$ (58/77) de éstas no mostraban datos de infertilidad por obstrucción tubárica. No se identificó ninguno de los otros microorganismos investigados.

El modelo de regresión logística (x25 grados de libertad $=15.4, p=0.009$ ), para establecer el potencial confusor de la edad, en relación con los antecedentes clínicos y microbiológicos en las mujeres con infertilidad, no mostró reducción en el riesgo de infección para el número de parejas sexuales (con y sin ajuste; $R M=2.96$ vs 2.95 , respectivamente; $p<0.05)$. Otra variable que resultó significativa en el modelo ajustado estableció la relación del concubinato en el riesgo de infección $(\mathrm{RM}=7.93, p<0.05)$. Por otra parte, se evidenció el efecto protector de la infección por Candida albicans y de Gardnerella vaginalis en las pacientes con infertilidad y con aislamiento de Chlamydia trachomatis ( $R M=0.49$ y 0.41 , respectivamente; $p<0.05)$.

Por otra parte, $64.9 \%$ de las pacientes con infección mencionaron gestaciones previas $(50 / 77)$, de las cuales $9.1 \%$ tuvieron gestaciones con productos menores de 20 semanas; también se preguntó sobre los antecedentes de abortos y $31.2 \%$ respondieron haber tenido al menos uno, incluso una de las pacientes refirió haber tenido ocho.

Finalmente, se realizaron las revisiones ginecológicas para evaluar las alteraciones físicas del cérvix; en $66.2 \%$ el aislamiento se obtuvo de las pacientes con estructura normal, y sólo en $33.8 \%$ en las pacientes con anormalidades. Se evidenció que la probabilidad para identificar a $C$ trachomatis cuando hubo ectropión fue de 2.7 veces. Durante la revisión también se observó descarga vaginal en $64.9 \%$ de las pacientes con infección, de las cuales $28 \%$ (14/50) refirió dolor al momento de la auscultación abdominal (cuadro II).

\section{Discusión}

Uno de los patógenos que participa de manera importante en el desarrollo de las infecciones genitales es Chlamydia trachomatis; la presencia de este agente in- 


\section{Cuadro I

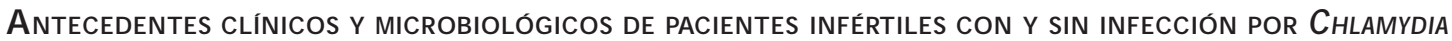 trachomatis. Instituto Nacional de Perinatología, Ciudad de México, 1998}

\begin{tabular}{|c|c|c|c|c|c|c|c|c|c|}
\hline \multirow[b]{2}{*}{ Variables } & \multicolumn{2}{|c|}{ Chlamydia positivo } & \multicolumn{2}{|c|}{ Chlamydia negativo } & \multirow[b]{2}{*}{$(\%)$} & \multirow[b]{2}{*}{ Valor $p$} & \multirow[b]{2}{*}{ RM (IC 95\%) } & \multirow{2}{*}{\multicolumn{2}{|c|}{ RMA (IC 95\%) }} \\
\hline & No. & Frec. & $(\%)$ & Frec. & & & & & \\
\hline Edad (años)* & & & & & & N.S & & & \\
\hline $18-24$ & 48 & 13 & 4.2 & 35 & 11.3 & & 1.0 & & 1.0 \\
\hline $25-34$ & 206 & 55 & 17.8 & 151 & 48.9 & & $0.98(0.48-1.99)$ & 0.32 & $(0.1-0.98)$ \\
\hline$>35$ & 55 & 9 & 2.9 & 46 & 14.9 & & $0.53 \quad(0.2-1.38)$ & 0.37 & $(0.15-0.95)$ \\
\hline Compañeros sexuales & & & & & & 0.008 & $2.96(1.22-7.5)$ & $2.95 *$ & $(1.16-7.49)$ \\
\hline 1 & 249 & 70 & 22.7 & 179 & 57.9 & & & & \\
\hline$>2$ & 60 & 7 & 2.3 & 53 & 17.1 & & & & \\
\hline
\end{tabular}

\begin{tabular}{lrrrrrrrrr} 
Estado civil* & \multicolumn{1}{c}{0.03} \\
\hline Soltera & 15 & 2 & 0.6 & 13 & 4.2 & 1.0 & 1.0 \\
\hline Casada & 247 & 58 & 18.8 & 189 & 61.2 & $1.99(0.44-9.08)$ & 1.76 & $(0.91-3.41)$ \\
\hline Concubinato & 47 & 17 & 5.5 & 30 & 9.7 & $3.68(0.37-36.28)$ & $7.93^{*}(0.96-65.71)$
\end{tabular}

\begin{tabular}{|c|c|c|c|c|c|c|c|c|}
\hline Métodos de control*ł & & & & & & & & \\
\hline Sin control & 184 & 39 & 13.8 & 145 & 51.2 & 1.0 & & 1.0 \\
\hline hormonal & 42 & 12 & 4.2 & 30 & 10.6 & $(0.7-3.18)$ & 1.95 & $(0.71-5.33)$ \\
\hline Local & 38 & 15 & 5.3 & 23 & 8.2 & $2.42(1.15-5.07)$ & 1.51 & $(0.47-4.87)$ \\
\hline $\mathrm{N}$ atural & 19 & 7 & 2.5 & 12 & 4.2 & $2.17 \quad(0.8-5.88)$ & 0.82 & $(0.26-2.59)$ \\
\hline
\end{tabular}

Infección cérvico-vaginal*\$

\begin{tabular}{|c|c|c|c|c|c|c|c|c|c|}
\hline thecelour cer vicu-vaginal & & & & & & TV.J & & & \\
\hline Sin aislamiento & 124 & 34 & 11.4 & 90 & 30.1 & & 1.0 & & 1.0 \\
\hline Candidiasis & 96 & 29 & 9.7 & 67 & 22.4 & & $1.15(0.64-2.07)$ & $0.49 *$ & $(0.23-1.01)$ \\
\hline Vaginosis bacteriana & 79 & 12 & 4 & 67 & 22.4 & & $0.47 \quad(0.23-0.98)$ & $0.41^{*}$ & $(0.19-0.86)$ \\
\hline Infertilidad por obstrucción tubárica & & & & & & N.S & $1.01 \quad(0.53-1.9)$ & 0.67 & $(0.33-1.34)$ \\
\hline Sí & 76 & 19 & 6.1 & 57 & 18.4 & & & & \\
\hline No & 233 & 58 & 18.8 & 175 & 56.6 & & & & \\
\hline
\end{tabular}

Notas: $n=309 ; R M=$ Razón de momios cruda; RMA = Razón de momios ajustada por edad, calculada por regresión logística, $p<0.05$ N S: no significante; N D: no determinado

\footnotetext{
* Tendencia de proporciones calculados por ji2; $p<0.05$ Valores perdidos

$\ddagger=8.4 \%$

$\S=3.23 \%$
}

feccioso frecuentemente no es identificada debido a que es una bacteria intracelular que requiere de los métodos diagnósticos de más alta sensibilidad y especificidad, los cuales son de alto costo o no están disponibles comercialmente; además, en la mayoría de las pacientes la infección cursa con un cuadro asintomático., 15-18

Basadas en la evidencia disponible, las implicaciones de la infección por $C$ trachomatis son en la actualidad tema de controversia. Mediante el empleo de cultivo celular la frecuencia del aislamiento observado en nuestro estudio en pacientes con infertilidad fue de $24.9 \%$ (77/309), esta prevalencia fue significativamen- te menor a lo reportado por otros autores. ${ }^{19,20} \mathrm{Sin}$ embargo, en los hallazgos de este estudio en relación con la infección de las mujeres infértiles comparados con los reportados en mujeres gestantes, ${ }^{21}$ en las inscritas en programas de planificación familiar y ginecológicos ${ }^{22}$ sugieren que la población femenina en general está en riesgo de adquirir la infección por $C$ trachomatis, contradiciendo las suposiciones de que esos grupos clínicos representan niveles de bajo riesgo. ${ }^{23}$

La tasa de infección por $C$ trachomatis orienta para establecer los requisitos generales en la prevención y el control de las enfermedades infecciosas por identi- 


\section{Cuadro II \\ Antecedentes ginecológicos de mujeres infértiles Con y sin infección por Chlam ydia Tracho matis. Instituto Nacional de Perinatología, Ciudad de México, 1998}

\begin{tabular}{|c|c|c|c|c|c|c|c|c|c|}
\hline \multirow[b]{2}{*}{ Variables } & \multicolumn{2}{|c|}{ Chlamydia positivo } & \multicolumn{2}{|c|}{ Chlamydia negativo } & \multirow[b]{2}{*}{$(\%)$} & \multirow[b]{2}{*}{ Valor $p$} & \multirow[b]{2}{*}{ RM (IC 95\%) } & \multirow{2}{*}{\multicolumn{2}{|c|}{ RMA (IC 95\%) }} \\
\hline & No. & Frec. & $(\%)$ & Frec. & & & & & \\
\hline Abortos* & & & & & & N.S & & & \\
\hline 0 & 203 & 53 & 17.2 & 150 & 48.5 & & 1.0 & & 1.0 \\
\hline 1 & 42 & 10 & 3.2 & 32 & 10.4 & & $0.88(0.41-1.92)$ & 2.08 & $(0.12-35.1)$ \\
\hline$>2$ & 64 & 14 & 4.5 & 50 & 16.2 & & $0.79(0.41-1.55)$ & & N.D. \\
\hline
\end{tabular}

Gestaciones previas*

N.S

\begin{tabular}{lrrrrrrrr}
\hline 0 & 125 & 27 & 8.7 & 98 & 31.7 & & 1.0 & 1.0 \\
\hline 1 & 37 & 7 & 2.3 & 30 & 9.7 & 0.85 & $(0.07-0.98)$ & N.D. \\
\hline$>2$ & 147 & 43 & 13.9 & 104 & 33.7 & 1.5 & $(1.28-1.76)$ & N.D.
\end{tabular}

Cérvix* N.S

\begin{tabular}{|c|c|c|c|c|c|c|c|c|c|c|}
\hline Normal & 237 & 51 & 16.5 & 186 & 60.2 & & & 1.0 & & 1.0 \\
\hline Ectropión & 28 & 12 & 3.9 & 16 & 5.2 & & 2.74 & $(1.22-6.16)$ & 1.76 & $(0.25-12.5)$ \\
\hline Friabilidad & 27 & 10 & 3.2 & 17 & 5.5 & & 2.15 & $(0.93-4.98)$ & & N.D. \\
\hline Eritema & 17 & 4 & 1.3 & 13 & 4.2 & & 1.12 & $(0.35-3.58)$ & & N.D. \\
\hline Secreción vaginal & & & & & & N.S & 1.07 & $(0.6-1.9)$ & 1.22 & $(0.67-2.22)$ \\
\hline Sí & 197 & 50 & 16.2 & 147 & 47.6 & & & & & \\
\hline No & 112 & 27 & 8.7 & 85 & 27.5 & & & & & \\
\hline Dolor & & & & & & N.S & 1.34 & $(0.64-2.79)$ & 0.99 & $(0.42-2.32)$ \\
\hline Sí & 47 & 14 & 4.5 & 33 & 10.7 & & & & & \\
\hline No & 262 & 63 & 20.4 & 199 & 64.4 & & & & & \\
\hline
\end{tabular}

Notas: $n=309 ; R M=$ Razón de momios cruda; RMA = Razón de momios ajustada por edad, calculada por regresión logística, $p<0.05$

N S: no significante; N D: no determinado

* Tendencia de proporciones calculados por ji2; $p<0.05$

ficación selectiva de los casos, es decir, la búsqueda de portadores en la población altamente prevalente, asintomática, asociada con una significativa morbilidad, y que relativamente pueden ser diagnosticadas pudiendo ser eficazmente tratables en la pareja sexual. ${ }^{24}$

En algunos países, la recomendación terapéutica está basada en la determinación de la infección en el respectivo grupo poblacional, con la aplicación del tratamiento correspondiente a la mujer positiva a $C$ trachomatis y a su pareja sexual, siempre y cuando la prevalencia observada exceda $10 \%{ }^{25}$ No obstante, nosotros dirigimos el análisis bivariado de la información, para identificar los factores de riesgo independientes para la infección en el grupo de mujeres infértiles. Los factores con alta significancia que fueron asociados con la infección por $C$ trachomatis son quizás el hallazgo más sorprendente. El tener al menos una pareja sexual, el estar casada o conviviendo en concubinato y el uso del DIU para el control prenatal pueden ser las diferencias clínicas particulares de este grupo de mujeres. La identificación de los factores de riesgo en este estudio, como en otros trabajos, puede ser atribuida a la uniformidad de esos factores en el grupo de mujeres estudiadas, y a la prevalencia de la infección; estos resultados sugieren que el uso del DIU deberá restringirse a aquella mujer no infectada. ${ }^{26}$ De acuerdo con otros estudios, los métodos de control de no barrera se han asociado independientemente con el aislamiento de $C$ trachomatis en un grupo de mujeres inscritas en programas de planificación familiar. ${ }^{27}$

Muchos de los predictores independientes de la infección, reportados por otros autores como compo- 
nentes esenciales de las estrategias de búsqueda e identificación, no fueron determinados en este estudio. Por ejemplo, el criterio de la edad al inicio de la vida sexual, y el número de parejas sexuales, basados en la identificación selectiva de las mujeres en algunos grupos de poblaciones, puede ser defendible, pero el efecto protector en el incremento de la edad en relación con la infección no fue observado. Sin embargo, estos factores han sido asociados con infección cervical en mujeres atendidas en clínicas de infecciones de transmisión sexual. ${ }^{28}$ En estudios realizados principalmente en los EUA, la edad de las pacientes infectadas oscila entre los 13 y 18 años, ${ }^{29}$ comparativamente, en nuestro estudio, el grupo con mayor frecuencia de aislamiento estuvo entre los 25 y 34 años de edad. Este comportamiento puede ser explicado con base en el inicio de la vida sexual activa a edades más tempranas, diferente a lo observado en las mujeres mexicanas. ${ }^{11}$

De los hábitos que modifican la flora vaginal, como son el comportamiento sexual, el patrón higiénico y la utilización del DIU, 90\% de las mujeres con infertilidad infectadas por $C$ trachomatis refirieron tener sólo una pareja sexual y de éstas, $75 \%$ eran casadas. Es quizás importante clarificar el comportamiento sexual de la pareja y la posibilidad de perpetuación de la infección en la mujer casada. Hernández y colaboradores (1999), ${ }^{30}$ realizaron una encuesta sobre las características del comportamiento sexual en hombres de la Ciudad de México, y describieron que 79.4\% de éstos eran monógamos (1047/1319 casos), 15\% con múltiples parejas y $6.1 \%$ con una pareja regular. El $72.8 \%$ de los hombres monógamos tuvieron relaciones sexuales con una pareja regular en la última semana, $21.6 \%$ el último mes y $3.8 \%$ en el último año; de estos individuos sólo 194 utilizaron condón en sus contactos sexuales. En cuanto al uso del DIU, de las 38 pacientes que emplearon el método, 15 presentaron infección por $C$ trachomatis $(\mathrm{RM}=2.42 ; p<0.01)$, lo que sugiere que el empleo del dispositivo puede ser un factor incidente en el desarrollo de la infección que refleja la introducción de la bacteria en el tracto genital durante la colocación del mismo. Los datos epidemiológicos en otros países sobre anticoncepción e infección por $C$ trachomatis reportan que el empleo de anticonceptivos orales está directamente asociado con un aumento en la tasa de colonización cervical, debido a que los estrógenos hormonales favorecen su crecimiento, ${ }^{31}$ sin embargo, Wolner y colaboradores (1990) ${ }^{32}$ determinaron un decremento en el riesgo de EPI por Chlamydia trachomatis con el uso de anticonceptivos orales. Las diferencias entre este estudio y los reportados en EUA posiblemente se deban a la frecuencia con las que se realizan los cambios del DIU y las revisiones periódicas en esas pacientes, ya que este método de anticoncepción aumenta el riesgo de EPI. La presencia de un cuerpo extraño produce un fenómeno inflamatorio con presencia de una gran cantidad de leucocitos polimorfonucleares y macrófagos; este microambiente puede permitir el crecimiento y mantenimiento de $C$ trachomatis. $^{33}$

La frecuencia de coinfección con otros microrganismos de transmisión sexual como Candida albicans $(37.7 \%)$ o Gardnerella vaginalis $(15.6 \%)$ fueron factores de riesgo independientes para la infección por $\mathrm{C}$ trachomatis, a diferencia de un estudio realizado con mujeres jamaiquinas que participaron en programas de planificación familiar, donde se observó una alta tasa de positividad por Chlamydia trachomatis asociada con candidiasis $(52.6 \%) .^{22}$

La infertilidad es la común complicación de la EPI; después de un episodio, aproximadamente 15\% de las mujeres tendrán como resultado la adhesión peritubal o la obstrucción tubárica; desafortunadamente, muchos de los casos de EPI por $C$ trachomatis son asintomáticos y la sola manifestación clínica es la infertilidad tubárica. ${ }^{29}$ En este estudio, 58 mujeres con aislamiento $(75.3 \%)$ no mostraron infertilidad por obstrucción tubárica. Estudios retrospectivos han demostrado cierta correlación entre la presencia de anticuerpos anti-Chlamydia trachomatis y la infertilidad por factor tubárico, sin embargo, en aproximadamente 20\% de las mujeres fértiles también se han observado altos títulos. ${ }^{34,35}$

En cuanto al número de gestaciones y abortos, los resultados mostraron que $64.9 \%$ de las pacientes con infección por Chlamydia asistían por causa de una infertilidad secundaria y sin abortos previos, mientras que $35.1 \%$ restante asistía por infertilidad primaria y abortos. Los resultados sobre el número de abortos indicaron que cuatro abortos o más es un factor sugestivo para la búsqueda intencionada de $C$ trachomatis en estas manifestaciones patológicas.

Aunque la infección del cérvix por $C$ trachomatis es la más común de las infecciones genitales de la mujer, los resultados de nuestro estudio demuestran que sólo $33.8 \%$ de las mujeres con aislamiento observaron anormalidad a la exploración física del cérvix. En esos exámenes, el ectropión y la friabilidad fueron frecuentemente observados y estos hallazgos estuvieron implicados con el desarrollo de la infección ( $R M=2.7$ y 2.2 , respectivamente). Cabe señalar que los estratos celulares del aparato genitourinario se recambian constantemente, lo que provoca la erosión (ectropión) y la hemorragia (friabilidad) del epitelio, y produce un fenómeno pro-inflamatorio. Aunada a esta condición, la predilección de $C$. trachomatis por el epitelio cilín- 
drico y cúbico, y en especial por las células basales y parabasales, ${ }^{36}$ debido al reconocimiento de los receptores glicosaminoglicanos (péptidos glicanos de heparán sulfato) presentes en la célula hospedera, posibilitan la infección, aunque se ha sugerido que la adherencia de estas bacterias es en otros sitios específicos, expresados en la proteína principal de la membrana externa. ${ }^{37}$

Uno de los aspectos más desalentadores para la determinación de la infección por C trachomatis es la falta de manifestaciones clínicas. Los síntomas referidos son prurito vulvar, disuria, dispareunia, aumento y alteración de la descarga vaginal, dolor abdominal, sangrado irregular y ardor poscoito. Nuestros resultados demuestran que $63.8 \%$ de las pacientes observaron leucorrea; de ellas $16.2 \%$ tuvieron infección por Chlamydia trachomatis, y sólo 18.2\% manifestaron signos de dolor. De acuerdo con otros estudios, la descarga vaginal y el dolor abdominal al examen ginecológico no se correlacionaron con la infección por $C$ trachomatis, a pesar de ser la más común de las manifestaciones urogenitales en otros grupos poblacionales. ${ }^{27,28,36}$

La frecuencia de aislamiento de $C$ trachomatis observada en las mujeres con infertilidad sugiere la búsqueda, identificación y tratamiento preventivo en este grupo de pacientes, que ya puede ser más efectivo, como medida de control, que en la población general. Nuestras observaciones afirman la necesidad para acceder a cada población clínica y evaluar por separado los patógenos presentes en las infecciones de transmisión sexual, para el diseño de programas de escrutinio. Este estudio confirma la eficacia del cultivo celular en la búsqueda de reactores positivos para $C$ trachomatis, aunque no considera el costo-beneficio de este método para que sea usado en México como prueba de tamizado. Finalmente, los estudios epidemiológicos son necesarios para establecer los tratamientos presuntivos, siendo la mejor aproximación en la prevención y control de la infección por Chlamydia trachomatis en países como México, donde la prevalencia es considerable y el presupuesto asignado para la salud es limitado.

\section{Referencias}

1. C ates W, W asserheit J. G enital chlamydial infections: Epidemiology and reproductive sequelae. Am J 0 bstet Gynecol 1991;164:1771-1781. 2. Stamm W E. Chlamydia trachomatis infections: Progress and problems.J Infect D is 1999:179(2 Suppl):380-383.

3. Faro S. Chlamydia trachomatis: Female pelvic infection. Am J 0 bstet Gynecol 1999;164:1767-1770.
4. Sieper J, Kingsley G. Recent advances in the pathogenesis of reactive arthritis. Immunol Today 1996;17:160-162.

5. Chernesky M, Luinstra K, Sellors J, Schachter J, Moncada J, C aul 0 et al. $C$ an serology diagnose upper genital tract Chlamydia trachomatis infection? Sex Trans Dis 1998;25:14-19.

6. Land JA, Evers JLH, Goossens J. How to use Chlamydia antibody testing in subfertility patients. Hum Reprod 1998;13:1094-1098.

7. Scholes D, Stergachis A, Ichikawa LE, Heidrich FE, Holmes KK, Stamm W E.Vaginal douching as a risk factor for cervical Chlamydia trachomatis infection. 0 bstet Gynecol 1998;91:993-997.

8. Handsfield $H$, Jasman L, Roberts P. C riteria for selective screening for Chlamydia trachomatis infections in women attending family planning clinics. J Am Med Assoc 1986;255:1730-1734.

9. Brunham R, Cheang M. Chlamydia trachomatis infertility and population growth in sub Saharan Africa. Sex Transm D is 1992;20:168-173.

10. Plummer F, N gugi R. Prostitutes and their clients in the epidemiology and control of sexually transmitted disease. En: Holmes KK, Mårdh P, Sparling P,W einser PE, Ed. Sexually transmitted disease. N ueva York (N Y): McG raw Hill, 1990:71-76.

11. Bustos-López HH,Vázquez-Juárez ME,A rredondo-G arcía JL, LiraPlacencia J, Beltrán-Zúñiga M, Guerra-Infante F. Prevalencia de Chlamydia trachomatis en pacientes con esterilidad y embarazos no complicados. Perinatol Reprod Hum 1995;9:227-234.

12. Ibarra-C amacho A, Sosa-C eja R, Lugo-de la Fuente G. Frecuencia de Chlamydia trachomatis en mujeres estériles. An Esc $\mathrm{N}$ ac Cienc Biol Mex 1991;35:153-159.

13. N arcio RML,Arredondo GJL, Zaldívar N A, Q uesnel GBC, C asanova $R G, G$ uerra IF et al. Etiología microbiana de la enfermedad inflamatoria pélvica leve y moderada (EIP). Ginecol 0 bstet Mex 1998;66:309-315. 14. Hernández-Méndez JT,Alonzo-Rojo H, Escamilla-Avilés E, A quinoSantiago C, D eleón-Rodríguez I, Jiménez-Escalante $Z$ et al. Microorganismos asociados con Chlamydia trachomatis aislados de pacientes con leucorrea. An Esc N ac Cien Biol Mex 2000;46:53-61. 15. Guerra-Infante FM, Flores-Medina S, López-Hurtado M, SosaGonzález IE,Arredondo-García JL. Evaluación de la sensibilidad y especificidad de tres reactivos de inmunofluorescencia directa para el diagnóstico de Chlamydia trachomatis. Ginecol 0 bstet Mex 1995;63: 368-373.

16. Hill CS. Molecular diagnostic testing for infectious diseases using TMA technology. Expert Rev Mol Diagn 2001;1:445-455.

17. Joyner JL, D ouglas JM, Foster M, Judson FN . Persistence of Chlamydia trachomatis infection detected by polymerase chain in untreated patients. Sex Transm D is 2002;29:196-200.

18. Lan J, Melgers I, Meijer CJ,W alboomers JM, Roosendaal R, Burger C et al. Prevalence and serovar distribution of asymptomatic cervical Chlamydia trachomatis as determinate by highly sensitive PCR. J C lin Microbiol 1995;33:3194-3197.

19. Luo M, Zhang L, X iao Y.The prevalence of Chlamydia trachomatis and ureaplasma urealyticum cervical infection in infertility women and the observation of therapeutic efficacy. Human Yi Ke Da Xue Xue Bao 1998; 23:44-46.

20. So bocinski Z, Szymanski W, Adamczak R, Ludwikowski G, Przeperki $M, G$ ruzka M. Evaluation of incidence of Chlamydia trachomatis infections among the group of infertile women diagnosed by laparoscopy, and based on properties of Chlamydia trachomatis in the cervical canal, peritoneal fluid and ovarian cyst puncture. G inekol Pol 2001;72: 224-227.

21. French JI, McG regor JA, D raper D, Parker R, McFee J. Gestational bleeding, bacterial vaginosis, and common reproductive tract infections: Risk for preterm birth and benefit of treatment. 0 bstet Gynecol 1999; 93:715-724.

22. D owe G, Smikle M, King SD, W ynter H, Frederick J, Hylton-Kong T. High prevalence of genital Chlamydia trachomatis infection in women 
presenting in different clinical setting in Jamaica: Implications for control strategies. Sex Transm Infect 1999;75:412-416.

23. D owe G, King SD. G enital Chlamydia trachomatis (serotypes D-K) infections in Jamaican commercial street sex workers. Genitourin Med 1997; 73:362-364.

24. Paavonen J, Eggert-Kruse W. Chlamydia trachomatis: Impact on human reproduction. Hum Reprod Update 1999;5:433-447.

25. Kacmar J, Cheh E, Montagno A, Peipert JF.A randomised trial of azithromycin versus amoxicillin for the treatment of Chlamydia trachomatis in pregnancy. Infect D is O bstet Gynecol 2001;9:197-202. 26. Ross JD. An update of pelvic inflammatory disease. Sex Transm Infect 2002;78:18-19.

27. Stergachis A, Scholes D, Heidrich FE, Sherer D M, H olmes KK, Stamm $W$ E. Selective screening for Chlamydia trachomatis infection in a primary care population of woman. Am J Epidemiol 1993;38:143-153.

28. Behets FM,W illiams Y, Braithwaite A, Hylton-Kong T, Ho offman IF, $D$ allabetta $G$ et al. Management of vaginal discharge in women treated at a Jamaican sexually transmitted disease clinic: Use of diagnostic algorithms versus laboratory testing. C lin Infect D is 1995;21:1450-1455. 29. Pearlman MD, McN eeley SG.A review of the microbiology immunology and clinical implication of Chlamydia trachomatis infections. 0 bstet Gynecol 1992;7:448-461.

30. Hernández-G irón C, Cruz-Valdez A, Q uitero-Trenado M, Peruga A, Hernández-A vila $M$ et al. C aracterísticas de comportamiento sexual en hombres de la Ciudad de México. Salud Publica Mex 1999;41:95-100.
31. Hart G. Risk profiles and epidemiologic interrelationships of sexually transmitted disease. Sex Transm D is 1993;20:126-136.

32. W olner-H anssen P, Eschenbach DA, Paavonen J, Kiviat N, Stevens CE, Critchloww $C$ et al. Decreased risk of symptomatic chlamydial pelvic inflammatory disease associated with oral contraceptive use. JAMA 1990; 263:54-59.

33. Guerra-Infante FM, López-Hurtado M. Mecanismos inespecíficos en la eliminación de Chlamydia trachomatis. I. Aspectos microbiológicos y fagocitosis. Perinatol Reprod Hum 1999;13:205-213.

34. Shafer MA. Enfermedades de transmisión sexual en adolescentes. En: Hansfiel HH, ed. Enfermedad inflamatoria pélvica. Epidemiología, etiología, tratamiento, complicaciones. N ueva York (N Y): Practice Publishing C 0, 1990; Suplemento del Hospital:8-12.

35. Centers for Disease Control. Pelvic inflammatory disease: Guidelines for prevention and management. MMW R Morb Mortal W kly Rep 1991; 40:1-25.

36. Addiss D G, Vaughn ML, G olubjatnikov R, Pfister J, Kurtycz DF, D avis JP. Chlamydia trachomatis infections in women attending urban midwestern family planning and community health clinics: Risk factors, selective screening and evaluation of non-culture techniques. Sex Transm D is 1990;17: 138-144.

37. Moulder JW. Interaction of Chlamydia and host cells in vitro. Microbiol Rev 1991;55:143-190. 